\title{
Yod
}

Revue des études hébraïques et juives

La littérature israélienne, miroir d'une société multiple

\section{La question de l'identité dans le roman policier israélien : le cas Ohayon}

Question of Identity in Detective Novels

הזהות ברומן הבלשילשאלת

\section{Patricia Azerad-Sitbon}

\section{(2) OpenEdition}

Journals

\section{Édition électronique}

URL : https://journals.openedition.org/yod/367

DOI : $10.4000 /$ yod. 367

ISSN : 2261-0200

\section{Éditeur}

INALCO

Édition imprimée

Date de publication : 1 octobre 2009

Pagination : 155-170

ISBN : 978-2-85831-178-1

ISSN : 0338-9316

\section{Référence électronique}

Patricia Azerad-Sitbon, « La question de l'identité dans le roman policier israélien : le cas Ohayon », Yod [En ligne], 14 | 2009, mis en ligne le 31 octobre 2011, consulté le 08 juillet 2021. URL : http:// journals.openedition.org/yod/367 ; DOI : https://doi.org/10.4000/yod.367

Ce document a été généré automatiquement le 8 juillet 2021.

\section{(ब) $(1)$ ( $)$}

Yod est mis à disposition selon les termes de la Licence Creative Commons Attribution - Pas d'Utilisation Commerciale 4.0 International. 


\title{
La question de l'identité dans le roman policier israélien : le cas Ohayon
}

\author{
Question of Identity in Detective Novels
}

הזהות ברומן הבלשילשאלת

\author{
Patricia Azerad-Sitbon
}

1 Pour traiter du sujet de l'identité dans le roman policier, sans aborder pour autant l'histoire de ce genre en général ni ses définitions, il est nécessaire de poser d'emblée le postulat qui sert de fondement à notre intervention: le roman policier est le miroir d'une société, car, d'une part, son intrigue se déroule toujours dans un temps et dans un espace définis et d'autre part son but est de saisir avec réalisme des sujets restés tabous. Aussi, choisir ce genre d'écriture revient-il à reconnaître pour le descripteur son appartenance à la société qu'il dépeint. De même, le destinataire va recevoir l'argument, qu'il l'accepte, le refuse ou qu'il y adhère de façon variable, c'est-à-dire quelle que soit la position qu'il adopte par rapport à cette société.

2 À travers ce sentiment d'appartenance, et quel que soit le point de vue du narrateur ou du lecteur, s'exprime donc la notion d'identité. Par ailleurs, le héros, « qu'il soit fictif ou réel [...] est le produit d'un discours ${ }^{1}$ ». Et à travers la figure du héros, dans notre cas le héros du roman policier, l'œuvre propose un parcours dans une histoire de « souffrance comme identité ${ }^{~}$ interrogeant cette identité par les dessous de l'histoire, c'est-à-dire par le contexte historique d'où sont issus les personnages de l'intrigue, mais aussi par l'épreuve du meurtre et de ses conséquences morales et politiques.

3 De plus, l'intérêt du roman policier israélien en tant que marqueur identitaire tient d'abord au fait que ce genre disparaît complètement en Israël de la création de l'État jusque dans les années $1980^{3}$ (on n'y trouve que des traductions d'auteurs étrangers, anglo-saxons en particulier), puis au fait que certaines mutations de la société israélienne (guerre des Six Jours en 1967 d'abord, mais surtout celles du Kippour en 
1973 et du Liban en 1982) créent des conditions dans lesquelles le genre policier va s'épanouir et exprimer une revendication de l'Israël du XXI siècle.

Batya Gour (1947-2005) est la première à relancer le genre, trouvant dans le roman policier un véhicule adapté non seulement au traitement littéraire de la situation politique de son pays, mais adapté également pour amener à «repenser Israël $»^{4}$ : conforter le fondement identitaire national face aux soubresauts politiques et sociaux.

Précisons cependant que les romans de Batya Gour sont toujours mis en parallèle avec ceux de Shulamit Lapid qui fait paraître dans la même période une série policière, la série des Lizie Badihi, héroïne journaliste dont le combat féministe occupe le premier plan de son œuvre.

6 Les écrits policiers de Batya Gour constituent une série de six romans dont le héros principal est le détective Michaël Ohayon ${ }^{5}$.

7 Comment la question de l'identité se trouve-t-elle mise en avant?

8 Par une trame toujours identique qui met en exergue trois aspects de l'œuvre :

- la toile de fond: lieu et groupe social où se déroule le meurtre : expression de l'identité collective ;

- le personnage du détective : Michaël Ohayon : expression de l'identité individuelle ;

- expression de l'identité idéologique: la confrontation entre les fondateurs, le crime et Michaël Ohayon, fils spirituel des fondateurs, rencontre de trois éléments que l'on peut aussi exprimer dans l'équation suivante: inconscient collectif / traumatisme / conscience individuelle, sachant que les romans policiers de Batya Gour se caractérisent par leur fondement psychologique et philosophique.

\section{La toile de fond du meurtre : miroir de l'identité collective qui prend plusieurs colorations}

\section{Miroir de l'identité par le lieu où se déroule l'action : inscription dans l'espace, appartenance à un lieu}

9 Le cadre spatio-temporel met en scène, sauf dans Meurtre au kibboutz, la ville de Jérusalem des années 1980 aux années 2000. Pourtant, Jérusalem n'est pas une ville pour le crime : en effet, elle ne correspond pas aux critères habituels du roman policier, à savoir une ville tentaculaire qui pervertit l'individu. C'est pourquoi le choix de Jérusalem est symbolique en tant qu' «espace porteur de sens ${ }^{6}$ ", espace de la ville éternelle, métonymie de la terre d'Israël (Sion), où l'on emmenait les enfants « afin de façonner leur identité nationale ${ }^{7} »$ : réception identitaire spirituelle et territoriale.

10 En ce qui concerne Meurtre au kibboutz, il n'est plus question de ville, mais de territoire, celui où se situe le kibboutz, celui où vit une communauté animée de l'idéologie des pères fondateurs. Espace protégé, où la perversion de l'homme est contraire à la ligne de conduite. Espace protégé qui ne peut qu'entraîner un meurtre à motivation idéologique : réception identitaire idéologique. Ainsi, des personnages qui intègrent le kibboutz dans des conditions douloureuses y voient leur rédemption comme Osnat, la victime de Meurtre au kibboutz :

"[Osnat] pensait que seule la vie au kibboutz pouvait procurer une liberté intérieure, un sentiment de sécurité et d'appartenance » (p. 29/478), 
ou encore comme l'aide-soignante d'origine orientale, employée par le kibboutz, qui y voit la seule solution pour sauver son fils qui se drogue.

11 Enfin, par le statut particulier de la ville, Jérusalem est le point de fixation des identités et des rivalités entre Israéliens et Palestiniens: réception identitaire politique. D'ailleurs, les premiers soupçons, suite à la découverte d'un crime, s'orientent toujours vers un coupable palestinien.

\section{Miroir de l'identité sociale par le choix du groupe incriminé : inscription dans une mémoire, appartenance à une communauté de destin}

Les groupes mis sur le devant de la scène sont dépeints comme formant une communauté privilégiée, dépositaire de l'ensemble des normes de comportements et des codes sociaux: psychanalystes dans Meurtre $d u$ samedi matin, professeurs d'université dans Meurtre à l'Université, membres du kibboutz dans Meurtre au kibboutz, musiciens de renommée mondiale dans Meurtre au philharmonique, monde des affaires dans Meurtre sur la route de Bethléem, producteurs de cinéma dans Meurtre en direct.

Ce sont des microsociétés dont les membres sont considérés comme les fondateurs de l'État, soit émigrés d'avant la création d'Israël, soit rescapés de la Shoah, et dont il était impossible, jusque-là, de remettre en cause les valeurs. L'identité tourne alors autour de deux notions : celle de héros et celle de victime. Par quel procédé ?

En mettant l'histoire au service de la mémoire : dans tous les romans, le détective se lie à une personne référente du groupe incriminé qui raconte l'arrivée douloureuse des migrants en Erets Israël, éclaire les blessures cachées des victimes, révèle les côtés douloureux des meurtriers. Ces histoires sont reçues par les lecteurs «comme des témoignages des souffrances endurées, susceptibles d'en renforcer ou d'en pérenniser la mémoire ${ }^{9}$ ». Ces événements sont perçus et utilisés comme "une communauté de destins» donc comme une identité commune. Ces souffrances sont celles des fondateurs de l'État ${ }^{10}$ et des rescapés de la Shoah.

15 La légitimité des émigrés et celle de l'idéologie sioniste sont justifiées par les difficultés rencontrées lors de leur installation dans le pays et le bien-fondé de leur acharnement basé sur une idéologie progressiste. «L'histoire dont les fondateurs sont porteurs met l'accent sur leur héroïsme, héroïsme de victimes qui font front aux conditions de vie dans leur pays d'origine [...], mais aussi parce qu'ils se sont battus jour après jour pour leur survie et pour la construction du pays ${ }^{11}$ ». Quant aux rescapés de la Shoah, ceux qui se sont laissés conduire "comme des moutons à l'abattoir", "comment les Juifs diasporiques ainsi perçus pouvaient-ils être donnés en exemple ${ }^{12} »$ alors que la Shoah a d'abord été niée par une idéologie sioniste aspirant à créer un État neuf pour un «Juif nouveau »? C'est parce que le procès Eichmann (1961) avec "les témoignages des survivants [qu'il] sollicite, introduit l'Holocauste dans la conscience collective israélienne, et par la suite, juive en diaspora. L'identité de victime se transforme dans le pays en celle d'accusateur, et, par cette évolution, les survivants, les « moutons » d'hier, s'élèvent au rang de héros ${ }^{13}$ ». La construction du pays et la Shoah servent de mémoire collective et de ciment identitaire, la souffrance vécue par ces épreuves sert de «mémoire de solidarité dans l'affliction ${ }^{14}$ ». 


\section{L'identité s'exprime aussi par la Culture}

"Le décor, c'est le milieu; et tout milieu, notamment un intérieur domestique peut-être considéré comme l'expérience métonymique ou métaphorique d'un personnage ${ }^{15}$. protagonistes : appartements personnels d'une classe sociale aisée dans le quartier de Yemin Moshé, de Rehavia ou de la colonie allemande avec un intérieur garni de meubles de prix, de tableaux, de livres, de disques, cette métonymie diffuse le sentiment de culture et de savoir du milieu incriminé.

la communauté séfarade. Ainsi, lorsqu'il se retrouve en compagnie des membres de l'Institut psychanalytique tout de suite après le meurtre d'Éva Neidorf, Michaël Ohayon attribue l'animosité qu'il ressent à son encontre, non seulement aux préjugés envers la police, mais surtout au fait qu'il n'est pas lui-même "d'origine européenne » (Meurtre du samedi matin, p. 29/53) ${ }^{16}$.

19

Dans Meurtre sur la route de Bethléem, les différences ethniques sont exacerbées et se développent selon divers points de vue, économiques et culturels comme l'exprime la mère du meurtrier :

"Ils sont jaloux, c'est tout. ... IJaloux de ce que nous sommes... La jalousie les ronge, parce que nous [la famille Benech] sommes des gens cultivés et modernes alors qu'eux sont restés des primitifs. Ils sont parfaitement conscients, oui, oui, ils savent très bien que nous valons plus qu'eux» (p. 254//347),

ou dans des propos plus extrêmes encore par lesquels ce même personnage évoque des différences biologiques:

"On n'assassine pas dans n'importe quelle famille... Et, dans ce quartier, dans cette rue... voyez sur qui c'est tombé... Parfois... C'est dans les gènes... Il y a ... il y a ceux qui ont des bons gènes et ceux qui... les Schwartz ${ }^{17}$, quoi.. ...]. Ici, en Israël, il y a beaucoup d'Orientaux, et, comment dire, ce sont des gens...[...]. Le problème, c'est que dans cette rue, il y a trop de basanés, c'est tout.

- Maman!

- Tu veux que je le formule autrement? Trop de gens d'Afrique du Nord? Bon, eh bien, d'accord: à cause de tous ces gens d'Afrique du Nord, le niveau de la rue et du quartier et... de tout le pays d'ailleurs, a énormément baissé. Nous ne nous attendions pas à cela, franchement, nous n'avons pas été habitués... (p. 228/306).

Ces préjugés seront contrés par l'héritier de cette culture, le détective Michaël Ohayon, mais cette fois dans le rapport Séfarades / Arabes, comme nous le verrons plus loin.

\section{La Morale et les valeurs}

21 Tout le groupe social bouleversé par le crime, dont font partie les victimes et les meurtriers, est porteur de morale qui se traduit par leur attachement à une éthique, même dans le cas des meurtriers. Ainsi dans Meurtre du samedi matin, Joe Linder, un psychanalyste frustré par la victime, s'interroge-t-il :

"Qu'avait-elle donc fait pour s'attirer la haine d'une personne appartenant à un milieu qui, plus que tout autre, incarnait la loi et l'ordre?» (p. 60-61/109-111). 
ou, à propos de Touvia Shaï, le meurtrier du professeur Tirosh dans Meurtre à l'Université, il est dit :

"Touvia Shaï est un homme bien, foncièrement honnête ", (p. 84/122).

Et dans Meurtre au kibboutz, Yehuda Nahari, le supérieur temporaire de notre commissaire «ne consentait à fournir aide et directives que lorsque Michaël avouait pratiquement que s'ils piétinaient, c'était à cause de leur ignorance du milieu. Nahari se lançait alors dans des discours sur le kibboutz et ses valeurs » (p. 153/234).

C'est pourquoi, chez Batya Gour, le mobile du crime, le " pourquoi cela est arrivé » est plus important que le " comment ", c'est-à-dire la reconstitution de la scène fatale. La motivation tient une place importante, car « au-delà de la psychologie, il y a, en fait une question de morale : si nous voulons comprendre les motifs d'une action [...], c'est parce que nous en avons besoin pour porter un jugement moral sur cette action [...]. Le personnage apparait comme l'incarnation d'une conception de la personne et comme la mise en œuvre d'un exercice de morale ${ }^{18}$. Ainsi, les victimes sont tuées parce qu'elles veulent dénoncer un manquement moral; et les meurtriers deviennent meurtriers pour dissimuler un contournement moral. Par exemple, en Dina Silver, la meurtrière dans Meurtre du samedi matin, est dénoncée la tentative de dissimulation d'un concept déontologique fondamental de la profession, celui de ne pas avoir de relations avec son patient. Dans Meurtre à l'Université, l'homicide de Shaül Tirosh sur la personne de son étudiant, Ido Doudaï, s'explique par la tentative de dissimulation du concept moral « tu ne voleras point ", puisqu'il s'agit ici du vol de l'œuvre d'autrui, tandis que pour Touvia Shaï, le meurtrier du professeur Tirosh, celui à qui il vouait un culte, aucune norme morale ne peut contenir l'essence de l'Art pour laquelle il a tué, son seul absolu, son idéal, sa raison de vivre. Dans Meurtre au kibboutz, Dvorka, la vétérante et meurtrière tente de reprendre en main le kibboutz en effervescence en exhibant sa dernière carte, un ouvrage dans lequel sont consignés les principes des fondateurs du kibboutz, une sorte de «Bible », le Kehilatenou, dont elle lit un passage où il est question de sacrifice et de foi.

Les meurtriers de Batya Gour ont des exigences de perfection, souhaitent maintenir un état où la pérennité des rôles est la seule garantie de stabilité : leur groupe social est, sans conteste, le détenteur des valeurs universelles, rhétorique qui légitime un pouvoir identitaire justifiant une "mission civilisatrice». Et cette "mission civilisatrice " se concrétise à travers le personnage de Michaël Ohayon, modèle du nouveau Juif auquel s'ajoutera un autre rapport à l'Autre.

\section{Le personnage du détective. Expression de l'identité individuelle : le témoin ambassadeur}

Et notre détective M. Ohayon, va se conformer à ce modèle, «se fondre dans le creuset", comme il n'aura de cesse de le répéter d'une manière ou d'une autre. Comment? En adoptant tous les signes extérieurs et intérieurs du nouvel homme juif, lui le héros d'origine séfarade, né en 1948 (il émigre en Israël en 1951) suggérant par là même la réussite du sionisme, la pérennité du modèle.

Par quels procédés? 


\section{Une image conforme au nouvel homme juif par le physique, le vestimentaire et le comportement.} reproduits dans la relation des Séfarades avec les Palestiniens. Il appartient alors à Michaël Ohayon de mener la "mission civilisatrice» en réagissant aux paroles et attitudes de racisme que symbolise le personnage de Dani Balilti, inspecteur séfarade caricatural : en s'y opposant, notre héros combat le comportement d'une communauté orientale non détentrice du modèle salvateur, dénie tout racisme idéologique du sionisme et ainsi, le modèle reste européen.

\section{La morale}

\section{Michaël Ohayon reprend à son compte
devenant lui-même référent. Comment?}

Ces valeurs morales, mises en pratique au quotidien dans sa relation aux autres et sa position de justicier, sont confrontées aux attitudes du monde qui l'entoure : il est le Bien face au Mal, la Vérité contre le Mensonge, il est la Référence aussi bien par rapport à ses collègues, qui tous recherchent son aval, que par rapport aux témoins à charge qui le réclament personnellement en raison de ses qualités particulières d'humanité et de compréhension de même que les criminels lors des interrogatoires :

"Dans l'affaire Bitan, le commissaire Ohayon a réussi à se substituer pour le suspect à une sorte d'autorité morale, il lui est apparu... comme quelqu'un qui détenait le pouvoir d'absolution. Notre longue expérience, expliqua [Shorer], nous a appris que tout être humain a besoin de justification morale, et que parfois, avec de la chance, un enqueteur 
spécifique devient, aux yeux de son suspect, une personne qui a le pouvoir de lui accorder le

pardon, la légitimité.» (Meurtre au philharmonique, p. 350/552) ${ }^{19}$.

La conjonction de ces trois aspects : symbolique (couleur bleue et homme idéal), social (culture), éthique (morale personnifiée), fait de Michaël Ohayon un mythe vers lequel tous les personnages convergent, suspects, criminels, collègues de travail, femmes de sa vie et son fils envers lequel il agit tel un «père marocain qui se comportait avec son rejeton comme un Polonais rescapé de l'Holocauste » (Meurtre à l'Université, p. 23/33).

Si ces aspects constituent la réussite du héros sioniste, le parcours initiatique du bon Israélien, il n'empêche que la dépression chronique dans laquelle Michaël Ohayon se débat apparaît comme une angoisse existentielle, le symbole de la crise identitaire posée par l'évolution de la société des fondateurs, la réponse au choc que provoque le meurtre au sein du groupe privilégié :

"Il était paralysé de terreur chaque fois qu'il envisageait l'intrusion du monde extérieur dans sa réalité » (Meurtre au philharmonique, pp. 84 et 86/135 et 138).

«Sa réalité » est celle d'une société sioniste conforme à l'idéologie confrontée à l'inacceptable, le crime d'un groupe modèle; son mal-être celui du malaise contemporain, de l'individualisme et perte de sens.

\section{La confrontation : I'identité idéologique ou l'innocence perdue}

\section{Le choc au sein du groupe}

Dans tous les microcosmes spécifiques où se déroulent les enquêtes, tous ces groupes sociaux dont il était impossible, jusque-là, de remettre en cause les valeurs, réagissent violemment à l'annonce du crime, comme si était révélée la «part obscure » du groupe : l'un souffre d'un ulcère à l'estomac, symbole somatique et silencieux des maux du kibboutz, l'autre ne peut faire face à la situation des territoires occupés tandis qu'un troisième prend sa revanche en quittant le kibboutz et qu'un quatrième se suicide.

\section{Les conséquences}

\section{Il s'en dégage deux constats :}

D'une part, est mise en relief l'implosion de la réalité sociale qui exprime par la violence (le meurtre) la dilution, l'éclatement et la perte de la signification globale des valeurs. Par l'acte criminel, les fondateurs de l'État peuvent-ils encore être référents? Mais en même temps, le crime s'explique par la volonté de protéger un certain ordre social, gardien d'une culture occidentale sacralisée, régie par des valeurs universelles et une idéologie que les épreuves ne peuvent annihiler. Les épreuves ont fait la force de cette élite qui représente l'image positive de la société tout entière, l'exemple à suivre. Et la crise engendrée dans ses assises, véritable séisme national, remet en question les fondements de cette élite et du modèle social. Pourtant, l'histoire du crime devient l'occasion d'une reprise en main, d'une reconquête de soi dans un monde bouleversé. Les fondateurs redeviennent « les rassembleurs » qui ne révèlent un conflit qu'au titre d'épreuve leur permettant in fine de se reconstituer, de réactiver le sentiment du «nous ». 
41 En d'autres termes, le rôle de ces classes privilégiées, en tant que flambeau de la société, n'est pas remis en cause. Seule une réflexion sur les déviances doit être engagée pour mettre fin aux soubresauts du pays.

D'autre part, Michaël Ohayon, d'origine orientale, produit de cette élite qui lui a transmis ses éléments de distinction sociale et d'identification subit le contrecoup de la crise de son modèle.

La dépression chronique du commissaire est le miroir des failles de cette société idéalisée par sa culture, ses valeurs et son idéologie, le miroir des épreuves et souffrances des fondateurs nécessaires pour parvenir à une réalisation complète, le parcours de légitimation identitaire comme le fut celui des fondateurs de l'État, symbole de la représentation nationale.

44 Batya Gour ne se contente pas, à travers sa série, d'enraciner l'identité israélienne par le sentiment de reconnaissance en une identité collective, individuelle et idéologique. Elle pose le questionnement de la survivance identitaire en assurant une relève par l'intermédiaire de deux personnages : le lieutenant de police Yaïr (qui apparaît à partir du quatrième roman Meurtre au philharmonique) et Youval, le fils de Michaël Ohayon.

Le premier, fils spirituel d'Ohayon, physiquement identique ${ }^{20}$, est enfant du moshav et ses méthodes d'investigation s'inspirent de la botanique et de l'agriculture conformément à une éducation basée sur le rapprochement de la nature dans l'idéologie pionnière du travail de la terre. Double symbole : Yaïr est la continuité du modèle légué par le commissaire, lui-même dépositaire de l'idéologie sioniste, et le symbole du retour à la nature, en d'autres termes retour au point de départ de l'idéologie fondatrice.

Quant à Youval, le fils biologique, il pose les questions de la société israélienne notamment sur le statut de l'armée, la guerre et le côtoiement de la mort, la nécessité de quitter le pays " par soif d'espace et de grand air ${ }^{21}$ ". Et c'est par lui que se clôt le dernier roman, non plus dans la souffrance, mais dans le questionnement. En effet, les dernières pages se concluent (sans aucun lien avec l'intrigue) par une discussion entre le père et le fils sur la légitimité du sionisme (pp. 370-374/422-426).

\section{Conclusion}

Ainsi, par les représentations symboliques du groupe social sans lequel les caractéristiques du héros, Michaël Ohayon, n'auraient pu exister, Batya Gour affirme la conception sioniste sous toutes ses facettes. Mais le réel présuppose de maîtriser les œuvres, les institutions et surtout les principes sur lesquels le monde commun est établi. C'est le message de cette auteure et le sens de son propos «Je suis une sioniste moderne $^{22} »$ : le sionisme reste l'expression identitaire d'Israël, mais il doit prendre en compte « l'autre parti ${ }^{23}$ » au regard de l'éthique idéologique. 


\section{BIBLIOGRAPHIE}

BENBASSA, Esther (2007), La Souffrance comme identité, Fayard, Paris.

CHARBIT, Denis (2007), Qu'est-ce que le sionisme?, Albin Michel, Paris.

GOUR, Batya (2000), Jérusalem, une leçon d'humilité, Gallimard, Paris.

GOUR, Batya, « Je suis une sioniste moderne », propos recueillis par Laure Leibovitz, Le Figaro, 24 août 2004, article imprimé en ligne sur www.bladi.net

GREILSAMMER, Ilan (sous la dir. de) (1994), Repenser Israël : morale et politique de l'État juif, Autrement, Paris.

HAMON, Philippe (1979), « Pour un statut sémiologique du personnage », in : Barthes Roland, Poétique du récit, Seuil, Paris.

MOLINO, Jean \& LAFHAIL-MOLINO, Raphaël (2003), Homo fabulator : théorie et analyse du récit, Leméac, Montréal ; Actes Sud, Monaco.

SHAVIT, Yaacov \& Zohar (1983), Ha-ballash ha-'ivri hozer: mivhar ha-sippur ha-ballashi mi-Palestina, Monitin Books, Tel Aviv.

\section{NOTES}

1. In : Philosophie magazine, février 2008, $\mathrm{n}^{\circ} 16$, p. 98.

2. Voir : Benbassa (2007).

3. Pour l'histoire du roman policier israélien voir : Shavit, Yaacov \& Zohar (1983).

4. Expression empruntée à Greilsammer Ilan (sous la dir. de) (1994).

5. Retsah be-shabbat ba-boqer (1988) (Meurtre du samedi matin : un crime psychanalytique) (1993).

Mavet ba-hug le-sifrut (1989) (Meurtre à l'Université : un crime littéraire) (1994).

Linah meshuttefet (1991) (Meurtre au kibboutz) (1995).

Ha-merhaq ha-nakhon : retsah musiqali (1996) (Meurtre au philharmonique) (1997).

Retsah ba-derekh bayt lehem (2001) (Meurtre sur la route de Bethléem) (2003).

Retsah, metsallemim (2004) (Meurtre en direct) (2006).

6. Gour, Batya (2000), Jérusalem, une leçon d'humilité, Gallimard, Paris.

7. Gour, Batya, Jérusalem,... op. cit., p. 47.

8. Dans la signalisation adoptée (p. 29/47), le premier chiffre correspond à la page de l'édition hébraïque, le deuxième à la page de l'édition française.

9. Benbassa Esther, La Souffrance..., op. cit.

10. Meurtre du samedi matin rappelle les conditions d'installation en Israël à la fin des années 30, la fuite face à la montée du nazisme en Europe et les difficultés quotidiennes pour les nouveaux arrivants sur la terre de Palestine d'alors, tandis que dans Meurtre à l'Université, le professeur Klein rappelle le passé par ses liens avec les résidents de Rosh Pina (pionniers arrivés en 1878 pour construire, sans aucune expérience, la première ferme agricole ou mochava). Dans Meurtre au kibboutz, l'histoire de la société pionnière des kibboutzim s'illustre par les vétérans Sroulké et Dvorka Harel, pilier de la communauté. Dans Meurtre au philharmonique, les rescapés de la Shoah sont au premier plan. On retrouve cette catégorie de personnages, confrontés à la communauté d'origine orientale, dans Meurtre sur la route de Bethléem.

11. Benbassa Esther, La Souffrance... , op.cit. pp. 171-172

12. Ibid, pp. 241-242. 
13. Ibid, p. 179.

14. L'exemple le plus frappant, parce que clairement exprimé, se voit dans le dernier livre Meurtre en direct. Le meurtrier avoue avoir perdu tout contrôle de lui-même au moment où sa victime compare ses exactions passées au sort que les nazis ont fait subir à sa mère (p. 359/409).

15. Voir : Hamon (1979).

16. Voir aussi ibid p. 134/252.

17. Signifie « noir » en yiddish. Désigne les Juifs qui ne sont pas d'origine ashkénaze.

18. Voir : Molino \& Lafhail-Molino (2003).

19. Voir aussi Meurtre à l'Université p. 202/289 ; Meurtre sur la route de Bethléem p. 40/56.

20. «il ressemble un peu à ce que tu [Michaël Ohayon] étais il y a plus de vingt ans. Un grand maigre, avec des yeux profonds et des sourcils épais, le genre qui plaît aux femmes, à part qu'il n'est pas... il n'a pas... il est moins complexe » (Meurtre au philharmonique, p. 291/465).

21. Voir : Charbit (2007).

22. Gour Batya, "Je suis une sioniste moderne », propos recueillis par Laure Leibovitz, Le Figaro, 24 août 2004, article imprimé en ligne sur www.bladi.net

23. Terme évoqué dans Meurtre en direct (p. 239/275) pour désigner les Palestiniens.

\section{RÉSUMÉS}

La question de l'identité dans le roman policier israélien. Batya Gur est le premier auteur à avoir relancé le roman policier en Israël, un style qui avait disparu de la scène littéraire depuis la création de l'État en 1948.

L'étendue de son travail se compose de six romans écrits entre 1988 et 2004 . Son héros, le commissaire Michael Ohayon, natif du Maroc, enquête sur certains groupes privilégiés de la société israélienne, dont les membres sont parmi les fondateurs de l'État. Un crime perpétré dans les rangs de ces microsociétés (psychanalystes, professeurs d'université, membres de kibboutz, musiciens, cinéastes), submerge ces groupes de référents et également leur fils spirituel, Michael Ohayon, et remet en question le fondement idéologique des Pères fondateurs. Cette enquête idéologique au sein des groupes sociaux élevés et d'un point de vue personnel (Michael Ohayon) offre un nouveau regard aux questions d'autodéfinition telles que « qui êtes-vous? Que voulonsnous être? » à une société confrontée à la diversification sociale et politique.

Batya Gur is the first author to revive the detective genre in Israel, a genre that had disappeared from the literary scene since the creation of the State of Israel in 1948.

Her body of detective works is made up of six novels written between 1988 and 2004. Her hero, Superintendent Michael Ohayon, native to Morocco, makes his investigations into privileged groups of Israeli society, whose members are among the State's founders.

Crime that is perpetrated in the ranks of those micro-societies (psychoanalysts, professors of University, kibbutz's members, musicians, film-makers), overwhelms these groups of Referents and also their spiritual son, Michael Ohayon, and calls into question the ideological foundation of the Founding Fathers.

This ideological investigation within the high social groups and within the own personal view (Michael Ohayon) gives a new mind sight to the self-defining questions of the "who are you? What do we want to be?" to a society confronted with political and social diversification. 
הרומן הבלשי נעלם מבימת הספרות הישראלית עם הקמת המדינה ובתיה גור היא הסופרת שהחזירה אותו לחיים. בין 1988 הפשע המתבצע בקבוצות נבחרות אלו (פסיכולוגים, מרצים באוניברסיטה, חברי קיבוץ, מוזיקאים ואנשי קולנוע) מעורר שאן

\section{INDEX}

Mots-clés : quête identitaire, roman policier, société israélienne, diversité culturelle, Gour Batya (1947-2005), Sépharades, Ashkenazes

מילות מפתח

גיוון תרבותי, סיפור בלשים, החיפוש אחר הזהות, ישראל, ספרות, המאה העשרים,:

Index chronologique : vingtième siècle

בתיה גור, החברה הישראלית, הספרדי, אשכנזי Index géographique : Israël

Thèmes : littérature

Keywords : Israel, literature, twentieth century, Israeli society, quest for identity, Gour Batya (1947-2005), Sephardic, Ashkenazi, cultural Diversity, detective story 